\title{
Suppression of the Focal Shift of Single-Mode Laser with a Miniature Laser Processing Head
}

\author{
Jean-Francois Bisson and Hiroshi Sako \\ Amada, 200 Ishida, Isehara-City, Kanagawa, 259-1196, Japan \\ E-mail: bisson@amada.co.jp
}

\begin{abstract}
We propose a miniature processing head for laser cutting of metal sheets. The thermally-induced focal shift is exacerbated at low values of the beam parameter product, but it is possible to arrange the optical elements such that the focal shift and beam waist variation cancel out. Collimating and focusing low-NA beams with short focal lenses make it possible to use standard plano-convex lenses without causing appreciable spherical aberrations, but the higher power density calls for lowabsorption optics in order to mitigate thermal aberrations. The use of millimeter-size optics will pave the way to lighter processing heads, and this will enable an increased cutting speed in contours at a reduced electrical consumption. It can thus be considered as an alternative to remote cutting or to the addition of local axes to conventional gantry systems.
\end{abstract}

DOI: $10.2961 /$ jlmn.2009.03.0005

Keywords: Single mode lasers, focal shift, thermal lensing, processing head, beam quality.

\section{Introduction}

Laser processing of metal sheets generally involves the focusing of high-average power laser radiation into a spot size of a few hundred micrometers. High-power fiber laser sources emitting radiation at around $1 \mu \mathrm{m}$ wavelength are increasingly used as an alternative to $\mathrm{CO}_{2}$ lasers [1-5]. Besides the smaller footprint and the convenience of fiber delivery, 1- $\mu \mathrm{m}$ lasers have a lower electrical consumption and a higher cutting speed than with $\mathrm{CO}_{2}$ laser due to the higher brightness and higher absorbance by the metallic target. However, the laser operation by itself accounts for less than half the total electrical consumption of a laser cutting machine; moreover, the cutting speed in contours is limited to about $25 \mathrm{~m} / \mathrm{min}$ in conventional machines [6], due to the use of relatively bulky laser cutting head, which prevents large accelerations of the cutting head.

Remote cutting [6] has been developed for cutting thin sheets at higher speed. The addition of local axes to the conventional linear drive has also been proposed [7]. However, the former requires metal vaporization and thus requires a large specific energy, which makes it actually slower than conventional fusion cutting, not mentioning the mitigated cutting quality. The latter is an expensive solution and requires a complex algorithm in order to harmonize the displacement of both sets of axes.

In this paper, we propose the miniaturization of the laser cutting head in order to increase the cutting speed of contours, increase the maximum acceleration, and reduce the electrical consumption. High-brightness lasers make it possible to reduce the beam diameter on a lens while still enabling the focusing to a small spot size with a reasonable working distance. However, when the power density is increased, the question arises as whether thermal effects, such as thermal lensing and aberrations, thermal fracture and optical damage, will not limit the applicability of this idea. In this paper, we analyze the possibilities and limitations of the design of a miniature cutting head. First, we present the basic scaling laws governing thermal lensing. Then we present an arrangement for the suppression of thermal lensing in quasi-single-mode lasers. Then, we discuss limitations arising from absorption in optical elements, i.e., thermal distortions, absorption in lenses and optical damage.

\section{Scaling laws of thermal lensing}

\subsection{Focal shift dependence with brightness and focal distance}

Lenses usually contain a small amount of impurities in the bulk material or in the antireflection coatings that absorb a fraction of the incident laser power and generate heat. Heat diffusion produces a parabolic temperature profile, which produces an additional contribution, $\Delta\left(\frac{1}{f}\right)_{T L}$, to the dioptric power of the lens, $1 / f$. Simple differentiation gives a focal shift directed toward the lens as: $\Delta z=f^{2} \Delta\left(\frac{1}{f}\right)_{T L}$. The intensity variation produced at the target by the focal shift also depends on the Rayleigh parameter, $z_{R}$ : $z_{R}=\frac{\operatorname{BPP} f^{2}}{r_{0}^{2}}$ where BPP is the beam parameter product and $r_{0}$ is the beam radius impinging on the lens. The normalized shift is thus given by:

$$
\frac{\Delta z}{z_{R}}=\Delta\left(\frac{1}{f}\right)_{T L} \frac{r_{0}^{2}}{\mathrm{BPP}}
$$


It does not depend on the focal length of the focusing lens and scales inversely with the BPP. Now, the actual scaling with the beam radius at the lens requires the knowledge of the dependence of $\Delta\left(\frac{1}{f}\right)_{T L}$ with the beam radius $r_{0}$.

\subsection{Scaling of dioptric power $\Delta\left(\frac{1}{f}\right)_{T L}$}

Thermal lensing contains several contributions that can be divided into two main categories: thermal lensing induced by the temperature profile and that induced by the heating of the lens. In the first category, we must consider thermal lensing induced by the thermo-optical coefficient, by photoelasticity and by thermal expansion. In the second category, we consider the change of refractive index and thermal expansion. The calculation requires the knowledge of the material properties and the temperature profile inside the optical material. We will compare the results for two sizes of optical elements, described in Table 1. We assume a uniform heat source distribution over some radius $r_{0}$, transverse heat flow and constant thermal conductivity and $\mathrm{d} n / \mathrm{d} T$ as a function of temperature. We use the parameters of fused silica, i.e., thermal conductivity $k=1.4 \mathrm{~W} / \mathrm{m}-\mathrm{K}$, $\mathrm{d} n / \mathrm{d} T=1.110^{-5} \mathrm{~K}^{-1}$ and thermal expansion coefficient $\alpha=5.5$ $10^{-7} \mathrm{~K}^{-1}$ and $n=1.5[8]$.

Table 1. Dimensions of conventional and miniature optics and parameters used for the calculations in this paper.

\begin{tabular}{|c|c|c|}
\hline Beam radius $\boldsymbol{r}_{0}(\mathbf{m m})$ & $\begin{array}{c}\text { Conventional } \\
\text { optics }\end{array}$ & $\begin{array}{c}\text { Miniature } \\
\text { optics }\end{array}$ \\
\hline Lens radius $\boldsymbol{r}_{1}(\mathbf{m m})$ & 10 & 0.25 \\
\hline Thickness $\boldsymbol{t}(\mathbf{m m})$ & 25 & 1 \\
\hline Focal distance $\boldsymbol{f}(\mathbf{m m})$ & 150 & 1 \\
\hline Power $\boldsymbol{P}(\mathbf{k W})$ & \multicolumn{2}{|c|}{1} \\
\hline $\begin{array}{c}\text { Heat transfer coefficient } \\
(\boldsymbol{h}) \mathbf{W} / \mathbf{c m}^{2}-\mathbf{K}\end{array}$ & \multicolumn{2}{|c|}{50} \\
\hline Absorption $\boldsymbol{\eta}_{\mathrm{h}}(\mathbf{p p m})$ & \multicolumn{2}{|c|}{} \\
\hline
\end{tabular}

The dioptric power arising from the parabolic index profile is given by [9]:

$$
\Delta\left(\frac{1}{f}\right)_{d n / d T}=\frac{P \eta_{h}}{2 \pi k r_{0}^{2}} \frac{\mathrm{d} n}{\mathrm{~d} T}
$$

The dioptric power arising from the change of refractive index caused by strain is given by [10]:

$\Delta\left(\frac{1}{f}\right)_{r}=\frac{P \eta_{h}}{16 \pi k r_{0}^{2}} \frac{n^{3} \chi_{r / \phi} \alpha}{(1-v)}$

where we have different contributions in the radial and azimuthal directions (thermal birefringence):

$\chi_{r}=p_{11}(1-7 v)+p_{12}(7-9 v)=1.45$

$\chi_{\phi}=p_{11}(3-5 v)+p_{12}(5-11 v)=1.10$

and $p_{i j}$ are the photo-elastic coefficient for the material (fused silica). The thermal deformation effect scales as [10]:
$\Delta\left(\frac{1}{f}\right)_{\alpha}=\frac{P \eta_{h}}{\pi k r_{0}^{2}}(n-1) \alpha$

The relative contributions from these three effects are shown in Table 2. Due to the very low thermal expansion of fused silica, the $\mathrm{d} n / \mathrm{d} T$ is the dominant factor (91\%). All three quantities are proportional to the power density, and the relative contribution does not depend on the power, beam size and lens diameters.

Table 2. Contributions of the temperature profile to thermal lensing.

\begin{tabular}{|c|c|c|c|}
\hline$\Delta\left(\frac{1}{f}\right)_{T L}$ & $\mathrm{~d} n / \mathrm{d} T$ & Photoelasticity & $\begin{array}{c}\text { Thermal defor- } \\
\text { mation }\end{array}$ \\
\hline Formula & $(2)$ & $(3)$ & $(4)$ \\
\hline $\begin{array}{c}\text { Conventional } \\
\text { optics }\left(\mathrm{m}^{-1}\right)\end{array}$ & $6.35 \times 10^{-4}$ & $\begin{array}{c}(r) 2.3 \times 10^{-5} \\
(\phi) 1.8 \times 10^{-5}\end{array}$ & $3.1 \times 10^{-5}$ \\
\hline $\begin{array}{c}\text { Miniature } \\
\text { optics }\left(\mathrm{m}^{-1}\right)\end{array}$ & 1 & $\begin{array}{c}(r) 0.037 \\
(\phi) 0.028\end{array}$ & 0.05 \\
\hline
\end{tabular}

The maximum temperature rise at the center of the lens is given by: $\Delta T_{\max }=g \frac{\eta_{h}}{4 \pi k} \frac{P}{t}$

where $g\left(k, h, \frac{r_{0}}{r_{1}}\right)=1+2 \ln \left(\frac{r_{1}}{r_{0}}\right)+2 \frac{k}{h r_{1}}$

In contrast to the temperature profile, it is almost independent of the beam diameter and merely depends on the total absorbed power. The temperature rises calculated from (56) are $1.3{ }^{\circ} \mathrm{C}$ and $11.5{ }^{\circ} \mathrm{C}$ for the conventional and miniature optics respectively. We can calculate the dioptric power caused by the temperature $\Delta T_{\max }$ as:

$\Delta\left(\frac{1}{f}\right)_{\Delta n}=g \frac{\eta_{h}}{4 \pi k} \frac{P}{t} \frac{1}{f} \frac{1}{n-1} \frac{\mathrm{d} n}{\mathrm{~d} T}$

The contribution from thermal expansion is given by:

$\Delta\left(\frac{1}{f}\right)_{\alpha}=g \frac{\eta_{h}}{4 \pi k} \frac{P}{t} \frac{1}{f} \alpha$

These two contributions are compared in Table 3. The refractive index change is the dominant factor $(>97 \%)$.

Table 3. Dioptric power caused from the homogeneous temperature rise.

\begin{tabular}{|c|c|c|}
\hline$\Delta(1 / f)_{T L}$ & $\Delta n$ & $\alpha$ \\
\hline Formula & $(7)$ & $(8)$ \\
\hline Proportions & 1 & 0.025 \\
\hline Conventional optics $\left(\mathrm{m}^{-1}\right)$ & $2.0 \times 10^{-4}$ & $4.9 \times 10^{-6}$ \\
\hline Miniature optics $\left(\mathrm{m}^{-1}\right)$ & $3.4 \times 10^{-2}$ & $8.5 \times 10^{-4}$ \\
\hline
\end{tabular}

The magnitudes of thermal lensing from the first and second categories are compared in Table 4. We see that the temperature profile plays a dominant role for both conventional and miniature optics, and it becomes increasingly dominant as the optics size is reduced. Therefore, it is justified to use equation (2) to estimate the thermal lensing in fused silica. Combining eq. (1-2), we find: 
$\frac{\Delta z}{Z_{R}}=\frac{P \eta_{h}}{2 \pi k} \frac{\mathrm{d} n}{\mathrm{~d} T} \frac{1}{\mathrm{BPP}}$

Eq. (9) is valid for flat-top beams with spherical symmetry. The order of magnitude is expected to be correct for other power distributions, including Gaussian.

In summary, the focal shift normalized to the Rayleigh parameter is independent of the focusing distance and the beam radius. From the viewpoint of thermal lensing, there is no penalty involved in using smaller optics, smaller beam radius and shorter focal lengths. However, the normalized focal shift is higher in single-mode lasers than in multimode lasers because it is inversely proportional to the BPP. These conclusions were experimentally confirmed. Hence, the use of single-mode lasers calls for innovative solutions in order to mitigate the focal shift.

Table 4. Contributions of temperature profile and temperature rise to the thermal lensing.

\begin{tabular}{|c|c|c|c|}
\hline$\Delta(1 / f)_{T L}$ & $\mathrm{~d} n / \mathrm{d} T$ & $\Delta n$ & $\begin{array}{c}\text { Proportions } \\
\mathrm{d} n / \mathrm{d} T: \Delta \mathrm{n}\end{array}$ \\
\hline Formula & $(2)$ & $(7)$ & $3: 1$ \\
\hline $\begin{array}{c}\text { Conventional } \\
\text { optics }\left(\mathrm{m}^{-1}\right)\end{array}$ & $6.35 \times 10^{-4}$ & $2.0 \times 10^{-4}$ & $30: 1$ \\
\hline $\begin{array}{c}\text { Miniature } \\
\text { optics }\left(\mathrm{m}^{-1}\right)\end{array}$ & 1 & $3.3 \times 10^{-2}$ & \\
\hline
\end{tabular}

\section{Suppression of the focal shift in single mode beams}

\subsection{Focusing a Gaussian beam with a lens ${ }^{1}$}

We define the 'beam waist', $\omega$, as the minimum radius of a Gaussian beam along its propagation. At all other locations, we use the notation $r$ for the beam radius. The conjugation formula of the waists of a single-mode Gaussian beam, by a lens of focal length $f$, is [11]:

$$
\frac{1}{z_{0}+z_{R}^{2} /\left(z_{0}-f\right)}+\frac{1}{z_{1}}=\frac{1}{f}
$$

and the magnification, $m$, is given by:

$$
m \equiv \frac{\omega_{1}}{\omega_{0}}=1 / \sqrt{\left(1-z_{0} / f\right)^{2}+\left(z_{R} / f\right)^{2}}
$$

where $z_{0}$ and $z_{1}$ are the distances of object and image waists from the lens and $z_{R}=\frac{\pi \omega_{0}^{2}}{\mathrm{M}^{2} \lambda}$ is the Rayleigh parameter in the object side. In the following, the $\mathrm{M}^{2}$ parameter is taken equal to one in order to keep the formalism simple. The formulae can be generalized to $\mathrm{M}^{2}$ values higher than 1 , by replacing ' $\lambda$ ' by ' $\lambda \times \mathrm{M}^{2}$ 'in all the formu-

\footnotetext{
${ }^{1}$ The fundamental mode emitted by a fiber laser more closely resembles a secant hyperbolic function [1], which decays more slowly than a Gaussian beam. In the conjugation formulae, the difference with the Gaussian fundamental beam can be accounted for by adjusting the value of the $\mathrm{M}^{2}$ parameter.
}

lae. For small values of the $Z_{R}$ such that $z_{R}{ }^{2}<<\left|z_{0}\left(z_{0}-f\right)\right|$, eq. (10-11) reduce to the conjugation formulae of geometrical optics:

$$
\begin{aligned}
& \frac{1}{z_{0}}+\frac{1}{z_{1}}=\frac{1}{f} \\
& m=\left|\frac{f}{f-z_{0}}\right|=\left|\frac{z_{1}}{z_{0}}\right|
\end{aligned}
$$

In contrast to geometrical optics, there exists a maximum distance at which one can image a Gaussian beam, Fig. 1. To do so, the object $\omega_{0}$ is placed behind the object focal plane of the lens, such that the position of the waist, $z_{1}$, is stationary with respect to a change of $f$ :

$$
\left.\frac{\mathrm{d} z_{1}}{\mathrm{~d} f}\right|_{z 1 \max }=0
$$

This distance $z_{1}$ is equal to the Rayleigh parameter in the image side: $Z_{1 \max }=Z_{R}=\frac{\pi \omega_{1}{ }^{2}}{\lambda}$. However, as seen in

Fig. 2, the waist $\omega_{1}$ monotonously decreases as the dioptric power of the lens increases around the stationary position. Hence, one cannot, by using a simple lens, cancel both the derivative of magnification and that of the waist position with respect to the focal length. Nevertheless, one can see that, at $z_{1}=z_{1 \max }$, the curvature of the wavefront increases everywhere with infinitesimal increase of thermal lensing, dashed curve, Fig. 3. The design of the processing head proposed herewith makes use of this property, which is absent when considering the laws of geometrical optics.

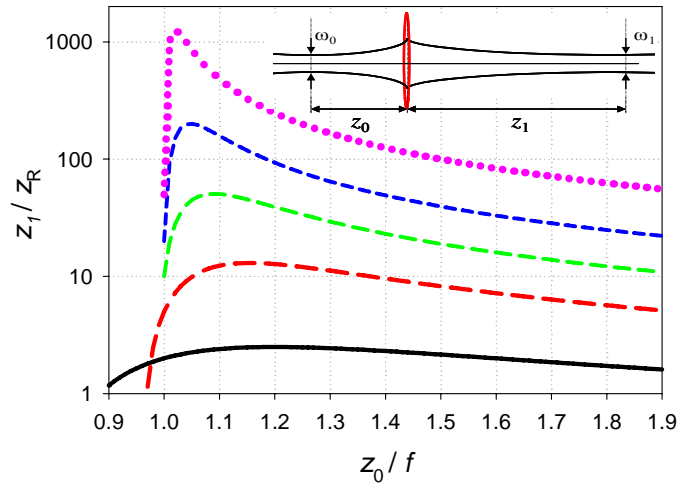

Fig. 1 Position of the waist of a Gaussian beam imaged by a lens in the paraxial approximation for various values of the Rayleigh length.

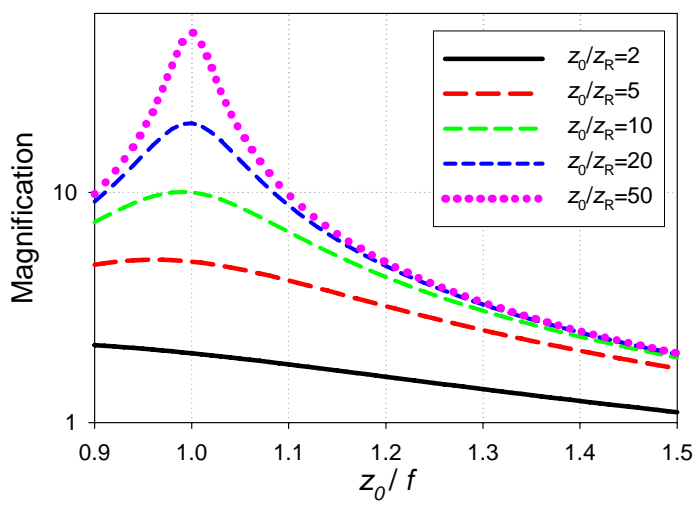

Fig. 2 Magnification factor of a Gaussian beam as a function object waist position $z_{0}$ for various values of the Rayleigh length. 


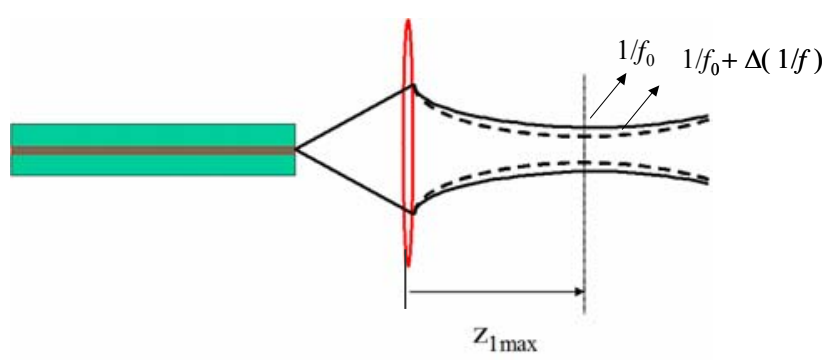

Fig. 3 Behavior of the beam caustic at the output of a SM optical fiber, solid to dash, for infinitesimal increase of dioptric power when $z_{1}=z_{1 \max }$. The waist does not shift to first order but its size decreases and the curvature of the wavefront increases.

\subsection{Stabilizing the beam caustic by using two lenses}

Consider the simple lens arrangement shown in Fig. 4. Let us assume that the collimating and focusing lenses are thin lenses, i.e., they leave the beam diameter unchanged but transform the wavefront radius according to the formula:

$\frac{1}{f}=\frac{1}{R_{1}}-\frac{1}{R_{2}}$

where $f$ is the focal length, $R_{1}$ and $R_{2}$ are the wavefront curvature at the entrance and exit surfaces of the lens, that take positive values for diverging beams. Coherent beam emerges from a single-mode fiber. The beam is characterized at any cross-section plane by its radius, $r$, and the wavefront radius of curvature, $R$. Let $\left(r_{1}, R_{1}\right),\left(r_{2}, R_{2}\right)$, $\left(r_{3}, R_{3}\right),\left(r_{4}, R_{4}\right)$ be the beam transverse radii and curvature radii of the Gaussian beam at positions 1, 2, 3 and 4 respectively, i.e. input and output interfaces of the collimating and focusing lenses respectively.

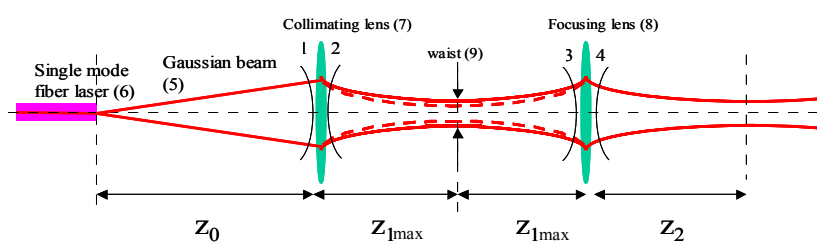

Fig. 4 Illustration of a thermal-lens-compensated processing head. The collimating (7) and focusing lenses (8) are placed symmetrically with respect to the waist (9). The solid line illustrates the beam caustic with stationary waist position (9). The dashed line illustrates the beam caustic for slightly increased value of the focusing power of collimating lens (7). Thermal lensing in the focusing lens (8) compensates for the increased curvature in entrance surface (3) to produce a stationary beam caustic after the focusing lens (8)

The focal distance $f_{1}$ of the collimating lens is selected such that the derivative of the waist position $z_{1}$ with respect to a change of $f_{1}$ is zero: $\mathrm{d}\left(z_{1}\right) / \mathrm{d}\left(f_{1}\right)=0$. The focusing lens is placed symmetrically with respect to the waist position (9), at a distance $2 z_{1 \max }$ from the collimating lens. Hence, the beam radii at positions (1), (2), (3), and (4) are equal, i.e. $r_{1}=r_{2}=r_{3}=r_{4}=r$ and stationary with respect to a change of $f_{1}$. Thermal lensing causes a change in the convergence of the collimating lens, $\Delta\left(\frac{1}{f_{1}}\right)$. From eq. (15), this causes an in- creased wavefront curvature $\Delta\left(1 / R_{2}\right)$ emerging from the collimating lens:

$$
\Delta\left(\frac{1}{R_{2}}\right)=-\Delta\left(\frac{1}{f_{1}}\right)
$$

The position of minimum waist $z_{1}$ remains unchanged to first order. By symmetry, the change of wavefront curvature at the entrance of the focusing lens (3) is the same as at position (2):

$$
\Delta\left(\frac{1}{R_{3}}\right)=-\Delta\left(\frac{1}{R_{2}}\right)
$$

At the focusing lens:: $\Delta\left(\frac{1}{R_{4}}\right)=\Delta\left(\frac{1}{R_{3}}\right)-\Delta\left(\frac{1}{f_{2}}\right)$, which, from (16-17), becomes:

$$
\Delta\left(\frac{1}{R_{4}}\right)=\Delta\left(\frac{1}{f_{1}}\right)-\Delta\left(\frac{1}{f_{2}}\right)
$$

Hence, thermal lensing taking place inside the focusing lens compensates the change of wavefront curvature taking place inside the collimating lens. Since the power density on both lenses is the same and the same material and similar thicknesses of lens material are used, then similar amount of thermal lensing should take place, i.e., $\Delta\left(\frac{1}{f_{2}}\right)=\Delta\left(\frac{1}{f_{1}}\right)$, and $\Delta\left(\frac{1}{R_{4}}\right)=0$. Therefore, both the beam radius $r$ and the radius of curvature $R_{4}$ are stationary at position 4 and the beam caustic after the focusing lens is stationary at every plane after the focusing lens. For this design, there is no restriction on the value of the focal length of the focusing lens.

In summary, focusing the fiber laser radiation onto a small spot size requires an optical system generally composed of two parts: the collimating lens (CL) and the focusing lens (FL). The design guidelines are as follows:

1) the fiber output and CL are arranged such that the derivative of the waist position with respect to the focusing power of the CL vanishes. This position is called P1.

2) There exists a point P2 further down the optical path where the beam diameter is stationary with respect to an increase of the focusing power of the CL. The FL is placed at $\mathrm{P} 2$.

3) The increased wavefront curvature at P2 caused by thermal lensing in the CL is (partly or totally) compensated by the thermal lensing of the FO.

\subsection{Example}

We consider a pair of lenses such as that shown in Fig. 4. The parameters are shown in Table 5. 
Table 5 Parameters used in the example of compensated focusing head.

\begin{tabular}{|l|l|}
\hline Emission wavelength $(\mu \mathrm{m})$ & 1.0 \\
\hline $\mathrm{M}^{2}$ & 1.0 \\
\hline Fiber laser emission waist $(\mu \mathrm{m})$ & 10 \\
\hline Collimating lens focal length $(\mathrm{mm})$ & 7.5 \\
\hline Focusing lens focal length $(\mathrm{mm})$ & 56 \\
\hline Distance from fiber end to collimating lens $\left(\mathrm{z}_{0}\right)(\mathrm{mm})$ & 7.8 \\
\hline Optimal spacing between the lenses $\left(\mathrm{L}=2 \mathrm{z}_{1}\right)(\mathrm{mm})$ & 194 \\
\hline Beam diameter at the lenses $\left(2 r_{0}\right)(\mu \mathrm{m})$ & 496 \\
\hline $\begin{array}{l}\text { Distance from focusing lens to waist at work }\left(\mathrm{z}_{2}\right) \\
(\mathrm{mm})\end{array}$ & 69 \\
\hline Waist at the work $(\mu \mathrm{m})$ & 96 \\
\hline
\end{tabular}

For the calculation of the focus shift, we use: $\Delta\left(\frac{1}{f}\right)_{d n / d T}=\frac{P \eta_{h}}{2 \pi k r_{0}^{2}} \frac{\mathrm{d} n}{\mathrm{~d} T}$. Taking $\mathrm{P}=1 \mathrm{~kW}, \eta_{\mathrm{h}}=10^{-4}$, $r_{0}=248 \mu \mathrm{m}$, with the parameters of fused silica we get: $\Delta(1 / f)=1 / f_{\mathrm{TL}}=2 \mathrm{~m}^{-1}$. The position and size of the waist at the work are shown when the focusing power, $\Delta(1 / f)$ of the collimating and focusing lenses change by the same amount, in Fig. 5 and 6. We see that the focus shift from 0 $\mathrm{W}$ to $1 \mathrm{~kW}$ is less than $2 \mathrm{~mm}$ over that region and the waist size changes by less $4 \mu \mathrm{m}$ if the position of the fiber with respect to the lens is adjusted within better than $50 \mu \mathrm{m}$. If the distance between the two lenses is arbitrarily reduced from the optimal value 19.4 to $5.0 \mathrm{~cm}$, then the focus shift increases by a factor of four and the change of beam waist increases by a factor of two.

The lens misalignment in planes across the optical axis will introduce additional aberrations. We expect that a shift of the beam axis across the optical plane would not create significant additional amount of spherical aberrations in a plano-convex lens as long as the shift is small compared to the beam radius at the lens. The latter is at least a few hundred of microns, while we can mechanically adjust the lens and fiber axes within +/- 20 microns. Similarly, the tilt of the lens axis by an angle that is small compared to the output NA of the fiber will not contribute to a significant increase of aberrations. The alignment problem will be much less critical in the processing head than it would, for instance, when designing a coupling unit from single-mode to single-mode fibers.

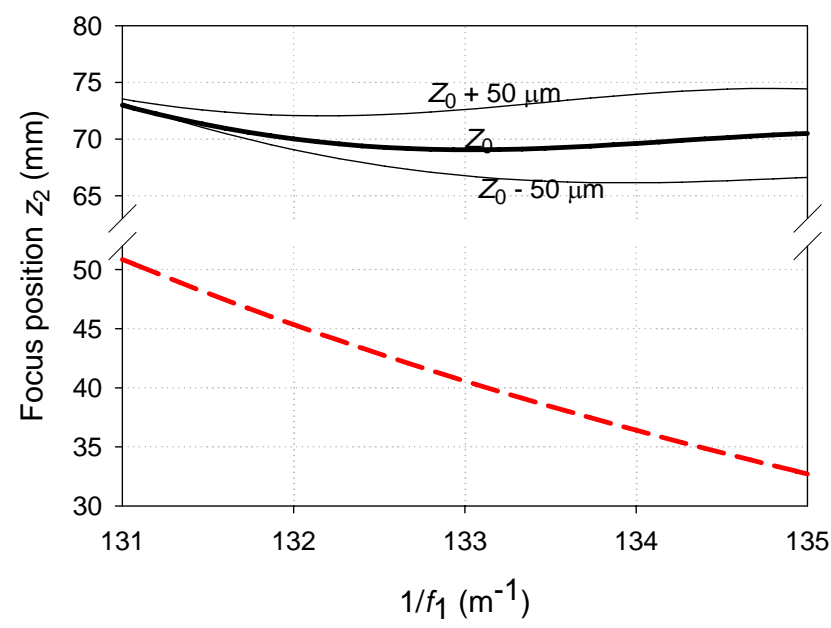

Fig. 5 Calculated focus shift at the work as a function of the focal power of the collimating lens for the parameters shown in Table 5. Changes $\Delta(1 / \mathrm{f})$ of the focusing and collimating lenses are assumed to be identical.
The dashed curve corresponds to a 5 -cm spacing between the lenses, and the solid curves correspond to the optimal spacing, $2 z_{1}=194 \mathrm{~mm}$. The light curves were obtained for the position of the fiber, $z_{0}$, shifted by $\pm 50 \mu \mathrm{m}$.

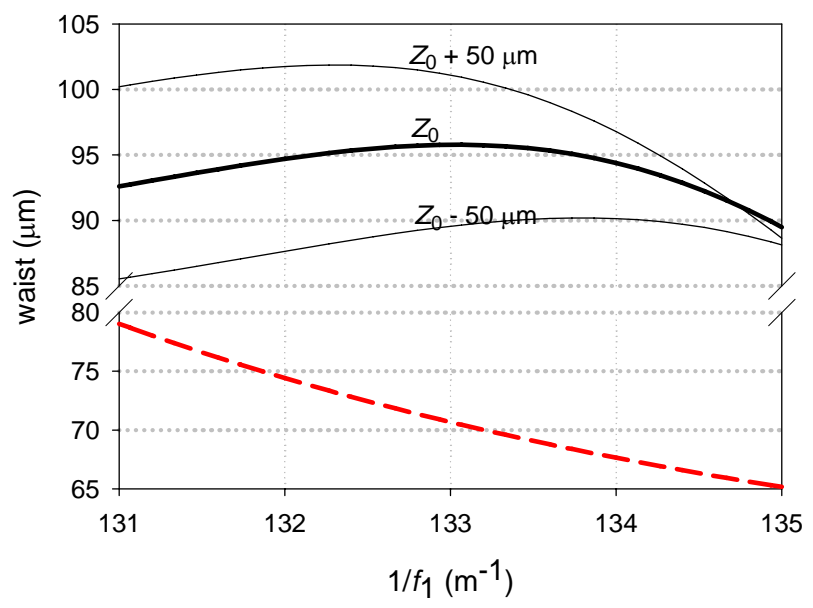

Fig. 6 Illustration of the calculated waist at the work as a function of the focal power of the collimating lens for the same conditions as in Fig. 5.

\section{Other considerations}

\subsection{Optical damage and spatter}

Optical damage limit of AR-coated optical elements is a thermal process triggered by absorbing defects. Despite its apparent simplicity, the degradation mechanism is not well understood even today, although thermal stress is believed to be an important factor $[12,13]$. It is a cumulative process where a damage spot gradually grows around an initiating defect. Nowadays, more than $1 \mathrm{MW} / \mathrm{cm}^{2}$ in continuous wave is routinely offered by optics makers [14], meaning that such optics can handle more than $1 \mathrm{~kW}$ power concentrated in a spot of roughly $300 \mu \mathrm{m}$ diameter. Hence, we think that the optical damage limit of optical elements should not prevent a significant reduction of beam size compared to existing designs.

Now, for miniature focusing optical head, the working distance would necessarily become shorter than for conventional optics. Then, the question arises as whether this would not cause additional maintenance problems due to spatters. The probability that spatters reach the lens and obstructs the beam is proportional to the solid angle of the beam at the lens viewed from the workpiece, which is proportional to the square of the numerical aperture (NA). Now, the spot size at the workpiece is adjusted to be similar to conventional head; this means that the resulting NA must be significantly smaller than conventional cutting heads due to the low BPP value of a quasi-single-mode beam. Hence, a lower probability of a spatter obstructing the beam is predicted. Nevertheless, the eventual spatter would dramatically impact on the focus shift; hence, a protecting window with a spatter-monitoring approach might be needed to protect the optics. The protecting window should itself be replaced on a regular basis and this could be achieved by moving the window laterally to expose a new part when needed. 


\subsection{Geometrical and thermal spherical aberrations}

Aberrations of plano-convex lenses in conventional optics is prohibitive for the coupling of single mode lasers. Hence, aspherical lenses, achromats or doublets are generally used for the coupling of single mode beams. However, the use of very low NA and short focal distances changes the state of affairs. The spot size caused by spherical aberration for a plano-convex lens made of fused silica at the infinite conjugate can be calculated from ref. [15] as:

$$
D_{S A} \approx \frac{1}{8 \sqrt{2}(n-1)^{2}} \frac{f}{\mathrm{No}^{3}} \approx 0.35 \frac{f}{\mathrm{No}^{3}}
$$

where $f$ is the focal distance and No is the f-number, defined as the ratio of focal distance to beam diameter. In comparison, the diffraction-limited spot size is given by:

$$
D_{D L}=\frac{2 \lambda}{\pi N A} \approx 0.64 \frac{\lambda}{N A}
$$

Considering the example in Table 1 , we find that the fnumbers are No $=7.5$ and No $=15$ for the conventional and miniature optics respectively. These values give a spot size caused by spherical aberration of $D_{S A}=124 \mu \mathrm{m}$ and $D_{S A}=0.8 \mu \mathrm{m}$ respectively. The respective diffractionlimited spot sizes are respectively $D_{D L}=10 \mu \mathrm{m}$ and $D_{D L}=21$ $\mu \mathrm{m}$ at $\lambda=1.1 \mu \mathrm{m}$. Hence, the use of plano-convex lenses is sufficient to conjugate the laser output to the workpiece without significant degradation of the $\mathrm{M}^{2}$ factor for the miniature optics, which is not the case when optics of conventional size is used. The use of simple plano-convex lenses simplifies the design. Moreover, the smaller beam diameter helps to minimize the thickness and absorption inside the optical elements, which contributes to minimizing thermal lensing.

In comparison, thermal aberrations arising from deviations from parabolic index profiles, present a serious impediment to the use of miniature optics because of the very steep temperature gradient that comes together with a small beam diameter. When considering thermal aberrations, the actual power distribution is important because the quartic terms in the temperature distribution play a dominant role. For Gaussian beams, the spot size contributed by thermal aberrations calculated from the second-order moment is [15]:

$$
D_{T H} \approx 0.45 \frac{\Gamma P \eta_{h}}{k} \frac{1}{N A}
$$

where $\Gamma=\frac{\mathrm{d} n}{\mathrm{~d} T}+(n-1) \alpha$. The dependence of the spot sizes calculated from eq. 19-21 as a function of absorbed power is shown in Figs. 7-8 for Gaussian beam impinging on the conventional and miniature size optics described in Table 1, respectively. For conventional optics, spherical aberrations play a dominant role, whereas for miniature optics, thermal aberrations dominate the diffraction-limited spot when the absorbed power exceeds a few hundred $\mathrm{mW}$. Hence, the use of miniature size optics opens the possibility of plano-convex lenses, but it is necessary that absorption and contamination at optical surfaces be kept at a very low level.

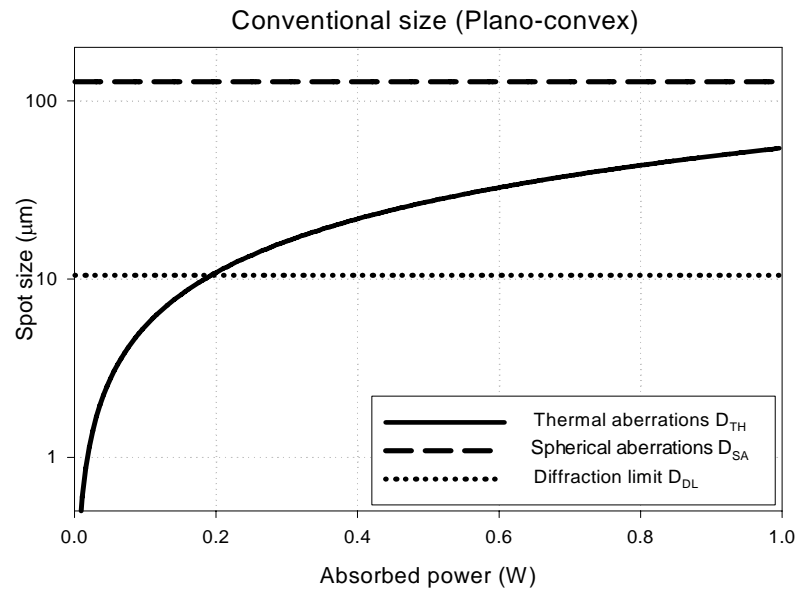

Fig. 7 Contributions to the spot size for thermal and intrinsic spherical aberrations and diffraction limited spot for conventional optics. Spherical aberrations dominate.

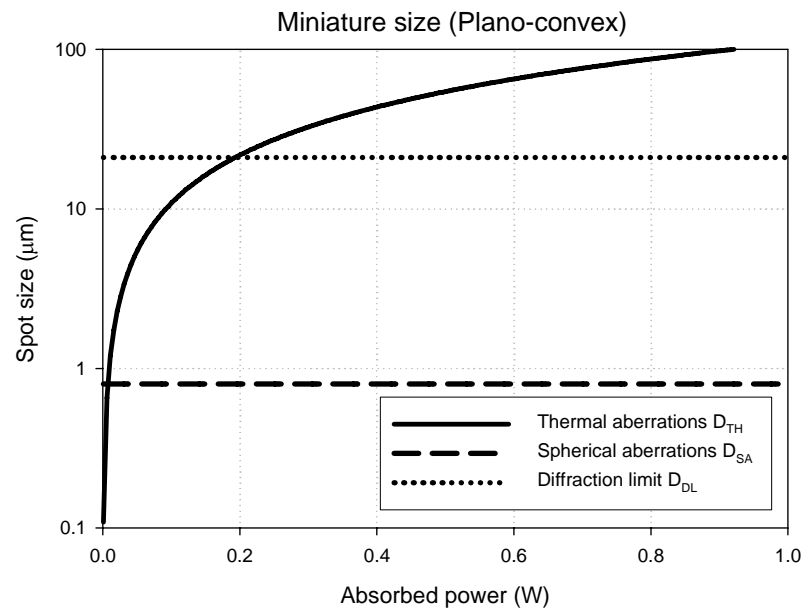

Fig. 8. Contributions to the spot size for thermal and intrinsic spherical aberrations and diffraction limited spot for miniature optics. The diffraction-limited spot is obtained up to a few hundred $\mathrm{mW}$ absorbed power, above which thermal aberrations dominate.

\section{Conclusion}

Miniaturization of the processing head is proposed as a means to reduce the electrical consumption and increase the cutting speed in contours. This proposal is justified by an analysis of the scaling of thermal lensing, which shows that the focal shift normalized to the Rayleigh parameter does depend neither on the focal length nor on the beam diameter. However it scales inversely with the BPP. A design was proposed that involves very small beam diameters and can also cancel the focal shift. Optical damage is not expected to be a limiting factor. The main limitation caused by the higher power density on the optical element was found to be thermal aberrations. Reduction of absorption and suppression of contamination are key factors in order to achieve miniature laser cutting head. We expect that its realization will enable significant gains of productivity and reduction of electrical consumption of laser cutting machines. 


\section{References}

[1] M. Blomqvist, M. Palsson, O. Blomster and G. Manneberg, "Fundamental mode fiber-to-fiber coupling at high power", Photonics West 2009, paper 7193-15.

[2] F. Abt, A. Hess, F. Dausinger, "Focusing of highpower single mode laser beams", ICALEO 2007, Laser Material Processing Conference, (2007), p.77.

[3] T. Himmer, T. Pinder, L. Morgenthal, E. Beyer, "High brightness lasers in cutting applications", ICALEO 2007, Laser Material Processing Conference, (2007), p.87.

[4] B. Wedel, "Focusing high brightness lasers- special requirements on laser processing heads", ICALEO 2007, Laser Material Processing Conference, (2007), p.83.

[5] O. Blomster, M. Palsson, S.-O. Roos, "Optics performance at high power levels", Proc. SPIE, vol. 6871, (2008), p. 68712B.

[6] M. Lutke, A. Mahrle, T. Himmer, L. Morgnthal and E. Beyer, "Remote cutting - A smart solution using the advantages of high brightness lasers", ICALEO 2008, Laser Material Processing Conference, (2008), p. 695.

[7] F. Bartels, A. Klotzbach, T. Schwarz and L. Morgenthal, "Studies in increasing the dynamic of cutting machines - under the aspect of using dynamic beam deflection optics", 4th International Workshop on Fiber Lasers, Nov. 5-6, (2008), Dresden.

[8] S. Ream, W. Zhang, G. Firestone, C. Walters, "Zinc sulphide optics for high-power laser applications", ICALEO 2007, Laser Material Processing Conference, (2007), p.903.

[9] H. Glur, R. Lavi, T. Graf, "Reduction of thermally induced lenses in Nd:YAG with low temperature", T., IEEE J. Quantum Electron., 40(5), (2004), p.499.

[10] W. Koechner, "Thermal lensing in a Nd:YAG laser rod', Appl. Opt., 9(11), (1970), p.2548.

[11] S. A. Self, "Focusing of spherical Gaussian beams“, Appl. Opt., 22(5), (1983), p.658.

[12] A.A. Manenkov, G. A. Matyushin, V.S. Nechitailo, A. M. prokhorov and A.S. Tsaprilov, "Nature of the cumulative effect in laser damage to optical materials", Sov. J. Quantum Electron., 13(12), (1983), p.1580.

[13] A. F. Stewart, "Diagnostic Methods for cw laser testing“, Laser-induced Damage in Optical Materials: 2003, Proc. SPIE vol. 5273, (2003), p.50.

[14] CVI-Melles-Griot, http://www.mellesgriot.com/, e.g. optical damage limit specified in terms of continuous wave power density.

[15] J. Penano, P. Sprangle, A. Ting, R. Fischer, B. Hafizi and P. Serafim, "Optical quality of high-power laser beams in lenses", J. Opt. Soc. Am. B, 26(3), (2009), p.503. 